\title{
Determination of Technetium by Laser Induced Photoacoustic Spectroscopy Coupled with a Wave-Length Shifter Method
}

\author{
By T. Fujita, T. Sekine, H. Hiraga, K. Yoshihara, A. Mutalib, R. Alberto*, and J. I. Kim** \\ Department of Chemistry, Faculty of Science, Tohoku University, Sendai 980, Japan \\ * Labor fïr Radiopharmazie, Paul Scherrer Institut, CH 5232 Villigen, Switzerland \\ ** Institut für Radiochemie, Technische Universität München, D-8046 Garching, Germany
}

(Received May 12, 1993)

Laser induced photoacoustic spectroscopy /

Technetium / PZT detection /

Hexakis(thiocyanato) technetate

\begin{abstract}
Laser induced photoacoustic spectroscopy (LPAS) was applied to the determination of ${ }^{99} \mathrm{Tc}$ in a solution, coupled with quantitative complexation of $\mathrm{Tc}$ by thiocyanate ion $\left(\mathrm{NCS}^{-}\right)$to form $\left[\mathrm{Tc}(\mathrm{IV})(\mathrm{NCS})_{6}\right]^{2-}$, which had an intense absorption peak at $500 \mathrm{~nm}$. Sensitivity of the quantitative detection could be improved down to a concentration of $10^{-9} \mathrm{~mol} \mathrm{dm}^{-3}$ which was one order of magnitude better than that for thiourea complex.
\end{abstract}

\section{Introduction}

The long-lived radioactive nuclide, ${ }^{99} \mathrm{Tc}$, is one of the most important fission products with high fission yields $(6.06 \%)$. Its long half life $\left(2.13 \times 10^{5} \mathrm{y}\right)$ and characteristic pure $\beta^{-}$emission make its determination difficult. The authors have tried to develop a specific determination by ${ }^{99} \mathrm{Tc}\left(\gamma, \gamma^{\prime}\right){ }^{99 \mathrm{~m}} \mathrm{Tc}$ nuclear excitation [1-3], as well as by laser induced photoacoustic spectroscopy (LPAS) coupled with quantitative a complexation of technetium with thiourea (tu) to form $\mathrm{Tc}(\mathrm{tu})_{6}^{3+}$ [4]. In the case of the LPAS method using $\mathrm{Tc}(\mathrm{tu})_{6}^{3+}$, which has an intense absorption peak at $488 \mathrm{~nm}$ with a molar extinction coefficient of $6400 \mathrm{dm}^{3} \mathrm{~mol}^{-1} \mathrm{~cm}^{-1}$ [4], a detection limit of ${ }^{99} \mathrm{Tc}$ could be obtained down to the order of $10^{-8}$ mol dm ${ }^{-3}$.

In this paper, the hexakis(thiocyanato)technetate(IV) ion ([Tc(NCS) $\left.)_{6}\right]^{2-}$ ) has been chosen to improve the determination limit, because its absorption peak has a molar extinction coefficient larger than that for $\mathrm{Tc}(\mathrm{tu})_{6}^{3+}$. This work has been undertaken to provide a basis for development of a new chemical speciation using LPAS.

\section{Experimental}

The LPAS system consists of a nitrogen gas laser (Laserphotonics Co., UV24), a dye laser (Laserphotonics Co., DL14), and a gated boxcar averager system
(Stanford Research Systems, SR250). The detection part which includes piezo-electric tranducers (PZT) (Tohoku Kinzoku Co.) was home-made, and operation details was given in a previous report [4].

A $2 \mathrm{ml}$ sample solution in a quartz cell $(1 \mathrm{~cm}$ path length) was submitted to LPAS measurements by excitation with monochromatic laser pulse. The wave length of the excitation beam was adjusted to $500 \mathrm{~nm}$ using Coumarin-481 dye material.

$\left[\mathrm{Tc}(\mathrm{NCS})_{6}\right]^{2-}$ was prepared by the method according to Crouthamel [6]. A solution of $1 \mathrm{M} \mathrm{NH}_{4} \mathrm{SCN}$ was added into a $4 \mathrm{M} \mathrm{H}_{2} \mathrm{SO}_{4}$ solution containing pertechnetate. Within $5 \mathrm{~min}$, during stirring, the color of the solution changed to red-violet. Then a 1.5 times larger volume of acetone was added to stabilize the complex. An aliquot of this stock solution was diluted with distilled water to give an appropriate concentration of technetium for the LPAS measurements. An optical absorption spectrum was measured with a spectrophotometer (Hitachi 50-100). The radioactivity of $\mathrm{Tc}$ was determined with a liquid scintillation counter (Aloka LSC-700).

\section{Results and discussion}

The molar extinction coefficient of $\left[\mathrm{Tc}(\mathrm{NCS})_{6}\right]^{2-}$ at $500 \mathrm{~nm}$ was obtained as $46900 \mathrm{dm}^{3} \mathrm{~mol}^{-1} \mathrm{~cm}^{-1}$ by optical absorption spectrophotometry, which was in good agreement with the reported value [6] in the presence of acetone. Fig. 1 shows change of absorbance at $500 \mathrm{~nm}$ after mixing a pertechnetate solution with $1 \mathrm{M} \mathrm{NH}_{4} \mathrm{SCN}$ in the presence of acetone. It confirmed quantitative formation of $\left[\mathrm{Tc}(\mathrm{NCS})_{6}\right]^{2-}$ and its stability was guaranteed for standing of up to one day. In the absence of acetone, the absorption spectrum gradually changed to give another technetium species which has an absorption maximum at $410 \mathrm{~nm}$, as shown in Fig. 2. This species could be attributed to $\left[\mathrm{Tc}(\mathrm{III})(\mathrm{NCS})_{6}\right]^{3-}$ produced by further reduction of $\left[\mathrm{Tc}(\mathrm{IV})(\mathrm{NCS})_{6}\right]^{2-}[7,8]$.

When the acetone containing solution was submitted to the LPAS measurement, gas evolution could be observed and the true photoacoustic signals were drastically disturbed by this cavitation. Degasfication 


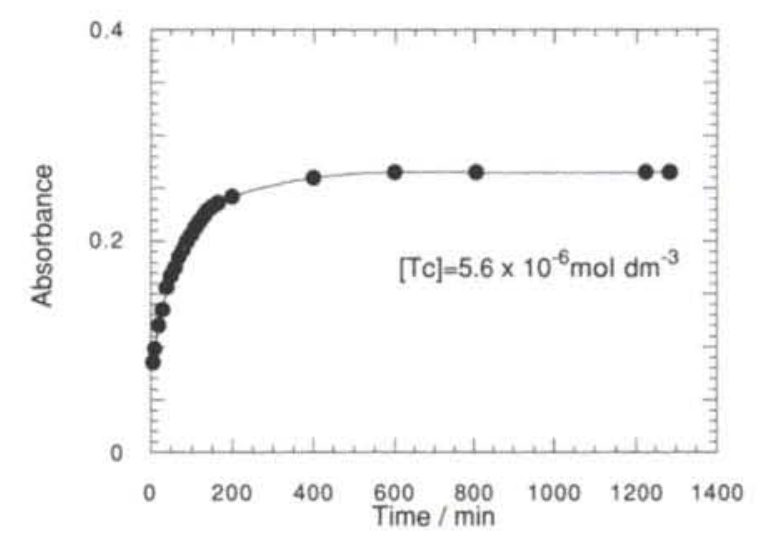

Fig. 1. The formation of $\left[\mathrm{Tc}(\mathrm{NCS})_{6}\right]^{2-}$ after mixing pertechnetate and $1 \mathrm{M} \mathrm{NH}_{4} \mathrm{SCN}$ solution in the presence of acetone.

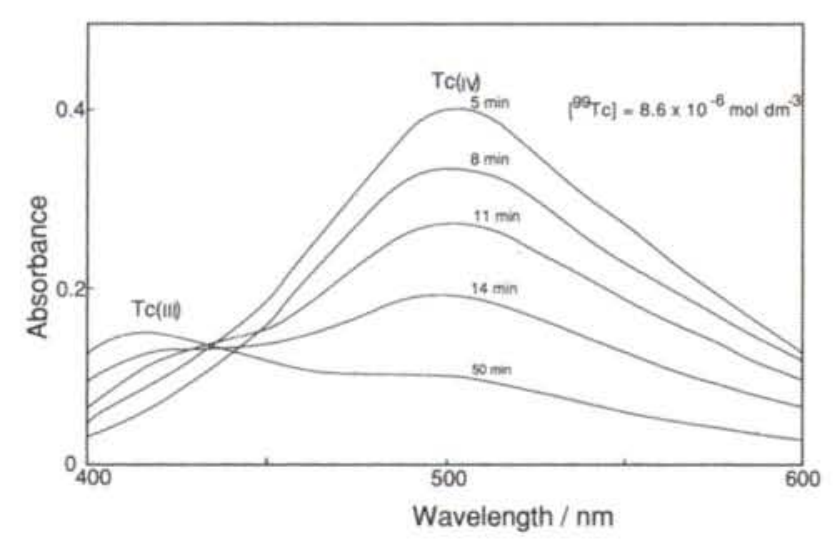

Fig. 2. The changes in absorption spectra of $\left[\mathrm{Tc}(\mathrm{NCS})_{6}\right]^{2-}$ in the absence of acetone.

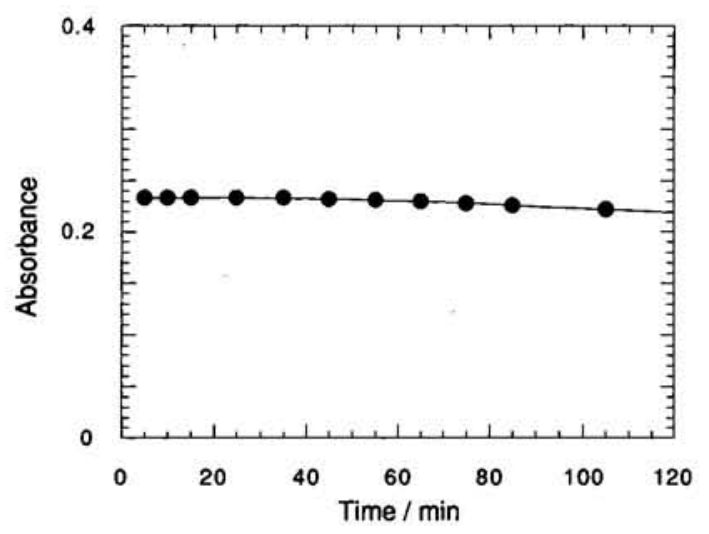

Fig. 3. The time dependence of absorbance of $\left[\mathrm{Tc}(\mathrm{NCS})_{6}\right]^{2-}$ when diluted with distilled water.

of the solution by ultrasonic treatment was not effective. Gas generation was not observed in the absence of acetone. Therefore, an aliquot of the stock solution of $\left[\mathrm{Tc}(\mathrm{NCS})_{6}\right]^{2-}$ stabilized by acetone was diluted with distilled water, and LPAS measurements were made immediately. The absorbance of the diluted solution at $500 \mathrm{~nm}$ slowly decreased with increasing time, as shown in Fig. 3. This change was slow enough to allow a quick measurement with $3 \mathrm{~min}$ after the sample

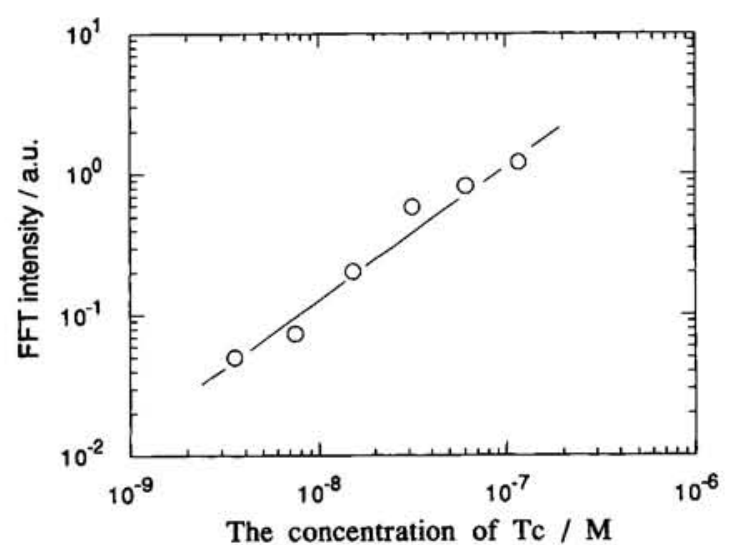

Fig. 4. The normalized FFT peak intensity as a function of the concentration of ${ }^{99} \mathrm{Tc}$.

preparation by increasing the laser pulse repetition to $20 \mathrm{~Hz}$ and by repeating 3 data averagings. Fast Fourrier Transfer (FFT) analysis was used for the averaged data to evaluate peak intensity [4].

The pressure wave, $p$, which is related to the intensity of photoacoustic signal, can be expressed by [9],

$$
p=\text { const. } \beta V_{a} E \alpha / C_{p}
$$

where $\beta$ is a thermal expansion coefficient, $E$ is pulsed laser energy, $C_{p}$ is specific heat at constant pressure, $V_{a}$ is acoustic velocity, and $\alpha$ is optical absorbance. $\alpha$ must be the value for a solute, $\varepsilon C$ ( $\varepsilon$ : molar extinction coefficient, $C$ : concentration) in a given medium. The net photoacoustic intensity of the solute can be obtained by subtracting the photoacoustic signal of the medium itself. Thus, the net acoustic pressure normalized to E can be directly related to the concentration of the solute, $\left[\mathrm{Tc}(\mathrm{NCS})_{6}\right]^{2-}$. In order to perform LPAS measurements for various concentrations of $\left[\mathrm{Tc}(\mathrm{NCS})_{6}\right]^{2-}$, different volumes of the stock solution containing acetone were diluted with distilled water to give a constant volume of $2 \mathrm{ml}$. This resulted in different compositions of water and acetone for each sample, which varies $\beta$ and $C_{p}$ in Equation (1). Acetone mixing would cause an increased background, because the increase of $\beta$ and decrease of $C_{p}$ are expected by addition of acetone. However, this effect was sufficiently small in the case of the reference solutions which changed the concentration of acetone up to $8 \%$. Almost constant photoacoustic signals were obtained, irrespective of change in the acetone concentration.

The FFT peak intensity normalized to the laser power is plotted against the concentration of ${ }^{99} \mathrm{Tc}$ in Fig. 4. A linear relationship could be obtained over the concentration range $10^{-7}-10^{-9} \mathrm{~mol} \mathrm{dm}^{-3}$ and the determination limit was improved by one order of magnitude better than the case using thiourea complex.

\section{Conclusion}

The determination limit was improved down to the order of $10^{-9} \mathrm{~mol} \mathrm{dm}^{-3}{ }^{99} \mathrm{Tc} ; 2 \mathrm{ml}$ of this solution is 
equivalent to about $440 \mathrm{mBq}$ of ${ }^{99} \mathrm{Tc}$. This value is comparable to that obtained by activation analysis [1].

\section{Acknowledgement}

This work was supported by the Grant-in-Aid of Ministry of Education, Culture and Science No. 03558022.

\section{References}

1. Sekine, T., Yoshihara, K., Németh, Zs., Lakosi, L., Veres, Á.: J. Radioanal. Nucl. Chem. Articles 130, 269 (1989).
2. Sekine, T., Yoshihara, K., Lakosi, L., Németh, Zs., Veres, Á.: Appl. Radiat. Isot. 42, 149 (1991).

3. Yagi, M., Sekine, T., Yoshihara, K.: J. Radioanal. Nucl. Chem. Lett. 155, 435 (1991).

4. Sekine, T., Hiraga, M., Fujita, T., Mutalib, A., Yoshihara, K.: J. Nucl. Sci. Technol, in press.

5. Hashimoto, K.: PhD Thesis, Tohoku University 1987.

6. Crouthamel, C. E.: Anal. Chem. 29, 1756 (1957).

7. Trop, H. S., Davison, A., Carey, G. H., DePamphilis, B. V., Jones, A. G., Davis, M. A.: J. Inorg. Nucl. Chem. 41, 271 (1979).

8. Trop, H. S., Davison, A., Jones, A. G., Davis, M. A., Szalda, D. J., Lippard, S. J.: Inorg. Chem. 19, 1105 (1980).

9. Patel, C. K. N., Tam, A. C.: Rev. Mod. Phys. 53, 517 (1981). 
